\title{
Factors affecting the recognition accuracy of facial expressions
}

\begin{abstract}
Facial expression recognition has many potential applications like detection of mental disorders, human behavior understanding and social interaction. The recognition accuracy of facial expressions is affected by many factors including, the intensity of the expressions, illumination effects, gender of face and image resolutions. This study investigates these factors and also presents an overview of some techniques who worked to resolve these issues.
\end{abstract}

Keywords: facial expressions, illumination effects, image resolution, expression intensity, accuracy
Volume I Issue 4 - 2017

\author{
Sajid Ali Khan \\ Department of Software Engineering, Foundation University \\ Rawalpindi campus, Pakistan
}

\begin{abstract}
Correspondence: Sajid Ali Khan, Department of Software Engineering, Foundation University Rawalpindi campus, Islamabad, Pakistan, Email sajidalibn@gmail.com
\end{abstract}

Received: August 19, 2017 | Published: November 16, 2017

\section{Introduction}

Facial expression recognition has many potential applications like detection of mental disorders, human behavior understanding and social interaction. The recognition accuracy of facial expressions is affected by many factors including, the intensity of the expressions, illumination effects, gender of face and image resolutions. This study investigates these factors and also presents an overview of some techniques who worked to resolve these issues. The human face possesses expressive ability ${ }^{1}$ and it provides one of the most natural, powerful and versatile means of affective mind state of humans. ${ }^{2}$ We use our facial expressions to provide important communicative cues and also express our emotions during social interaction. In order to perform analysis of expression in computer science, two main methodologies exists in literature i.e.

\section{a. Audio based methods and \\ b. Vision based methods.}

Expressions recognition through vision based methods is still the hottest research area due to the fact that expression can be emitted by a face without any sound. Vision based methods are generally divided into
a. Pre-processing,
b. Feature Extraction and
c. Expression classification.

Some pre-processing steps like face detection and normalization are performed on the input image in the first step. After face detection, the second and the most important step are to extract the face features from an image. Accuracy of any expressions recognition system greatly depends on how accurately features are extracted from given image. The process of features extraction is further classified into1) geometric-based feature extraction and 2) appearance-based feature extraction. Normally, location of the facial salient points and shapes of the facial components are used for geometric features extraction. Although geometric-based features are robust to illumination and other face variation but tracking of the facial salient points is very hard. Appearance changes of the face are utilized for appearance features extraction. One problem that associates with the appearancebased is that it produces extremely large number of features.

\section{Expression types}

Facial expressions can be divided mainly into six basic emotions, referred to as happy, sad, fear, disgust, surprise and anger. ${ }^{1,2}$ It is shown by different research community that the type of emotion expressed effects the accuracy of any expression recognition system.

\section{Expression intensity}

The expression recognition accuracy rate is dependent on intensity of emotional expressions. ${ }^{3,4}$ In general, the individuals recognized the expression more accuracy when the expression are more intense and less accurate in identifying subtle expressions.

\section{Illumination effects}

Illumination problems can be considered as a difficult problem to solve in both outdoor and indoor pattern matching. Two problems of textural based illumination handling in face image is very common i.e. due to increase in contrast, the textural values are changed during normalization. Secondly, it also increases the false acceptance rate because the distance between inter classes is minimized. ${ }^{5}$

\section{Image resolutions}

Many real world applications of face images required to work accurately on different image resolutions. However, literature studies revealed that these applications fail to achieve good accuracy rate when the size of image is reduced. ${ }^{6}$ Video conferencing, smart meeting and visual surveillance are some examples of such real time applications. This problem is tackled by very few studies in literature. Rizwan et al. ${ }^{6}$ first down sample the original image to produce the images with resolution and then pyramid of local binary pattern (PLBP) is applied to extract the features some facial points. Similarly, Khan et al. ${ }^{7}$ introduced a novel descriptor known as Weber Local Binary Image Cosine Transform (WLBI-CT) to handle the face images with different resolutions.

\section{Conclusion}

In this study, different factors affecting the accuracy of facial expression recognition has been investigated. Mostly the real world applications are sensitive to these factors. There are many algorithms presented in literature to solve these problems. It has been concluded 
that the conversion from spatial to frequency domain is the most convenient way to tackle some illumination and image resolution problems.

\section{Acknowledgments}

None.

\section{Conflicts of interest}

Author declares that there is no conflict of interest.

\section{References}

1. Darwin C. The Expression of the Emotions in Man and Animals. London: John Murray; 1872.

2. Ekman P. Emotion in the Human Face, Cambridge: Cambridge University Press; 1982.
3. Calder AJ, Young AW, Perrett DI, et al. Categorical perception of morphed facial expressions. Vision cognition. 1996;3(2):81-118.

4. Wells LJ, Gillespie SM, Rotshtein P. Identification of Emotional Facial Expressions: Effects of Expression, Intensity, and Sex on Eye Gaze. PLoS One. 2016;11(12):e0168307.

5. Shah JH, Sharif M, Raza M, et al. Robust Face Recognition Technique under Varying Illumination, Journal of Applied Research and Technology. 2015;13(1):97-105.

6. Khan RA, Meyer A, Konik H, et al. Framework for reliable, real-time facial expression recognition for low resolution images. Pattern Recognition Letters. 2013;34(10):1159-1168.

7. Khan SA, Hussain A, Usman M. Reliable facial expression recognition for multi-scale images using weber local binary image based cosine transform features. Multimedia Tools and Applications. 2017;1-33. 